\title{
Cefuroxime Axetil Loaded Gastroretentive Floating TabletsBased on Hydrophilic Polymers: Preparation and In Vitro Evaluation
}

\author{
Snehamayee Mohapatra ${ }^{*}$, Rajat KumarKar ${ }^{2}$, Debendra Kumar Mohapatra ${ }^{3}$, Sunit \\ Kumar Sahoo ${ }^{4}$ and Bhakti Bhusan Barik ${ }^{4}$ \\ ${ }^{1}$ Faculty of pharmacy; Sikshya O Anusandhan University; Bhubaneswar; 751003; Odisha - India.$^{2}$ Dadhichi \\ College of Pharmacy; Cuttack; 754002; Odisha - India. ${ }^{3}$ Jeypore College of Pharmacy; Jeypore; 764002; Odisha - \\ India. ${ }^{4}$ Utkal Univesity; Vanivihar; Bhubaneswar; 751004; Odisha - India
}

\begin{abstract}
The aim of this work was to study the formulation and in vitro characterization of hydro dynamically balanced floating matrix tablets using Cefuroxime axetil (CA) as model drug. Different excipients such as hydroxy propyl methyl cellulose (HPMC) K15M, E5LV (gelling agent), sodium bicarbonate (gas generating agent) and sodium lauryl sulfate (SLS) (solubility enhancer) were used in order to optimize the drug release profile as well as floating property. Decrease in release characteristics with high viscous polymer were observed due to increased gel strength, tortuosity and length of drug diffusion path. Significant difference $(p<0.5)$ in release rate was found at different concentration of SLS. The release mechanisms were explored and explained with zero order, first order, Higuchi, Korsmeyer and Hixson-Crowell equations. The release rate, extent and mechanism were governed by the content of polymer. The polymer content and amount of floating agent significantly affected the time required for $50 \%$ of drug release $\left(t_{50} \%\right)$, mean dissolution time (MDT), release rate constant, and diffusion exponent (n). Kinetic modeling of dissolution profile revealed that the drug release mechanism could range from diffusion controlled to case II transport, which was co-dominated by diffusion polymer erosion in the release mechanism.
\end{abstract}

Key words: Cefuroxime Axetil; HPMC; SLS; Release kinetics

\section{INTRODUCTION}

Rapid gastrointestinal transit could result in incomplete drug release from the device above the absorption region, leading to diminished efficacy of the administered dose (Iannuccelli et al. 1998). Therefore different approaches have been proposed to retain the dosage form in the stomach. These include bioadhesive systems (Santus, et al. 1997), swelling and expanding systems (Deshpande et al. 1996) and floating systems
(Menon et al. 1994). In some cases, gastro retention is achieved by concomitant administration of drugs or excipientswhich slow the motility of GIT (Moes 1994). Perhaps the most promising approach to achieving the gastro retention is that of creating a swelling or expanding system insitu. When the drug is formulated with a gel forming polymer such as semi synthetic derivative of cellulose, it swells in the gastric fluid with a bulk density less than one. It then remains buoyant and floats in the gastric

*Author for correspondence: dollypharma83@yahoo.com 
fluid, affecting a prolonged gastric residence time (GRT). This floating dosage form is known as a hydrodynamically balanced system (HBS (Ozdemir et al. 2000).Hydrodynamically balanced systems can remain in the gastric region for several hours and hence significantly prolong the gastric residence time of drugs. Prolonged gastric retention improves bioavailability, reduces drug waste, and improves solubility for drugs that are less soluble in a high $\mathrm{pH}$ environment of small intestine. It has some applications also for local drug delivery to the stomach and proximal part of small intestine (Ponchel and Irache 1998). Instead of having lot of potential benefits, floating drug delivery is associated with certain limitations. Drugs that irritate the gastric mucosa, multiple absorption sites in the gastrointestinal tract, undergo significant first pass metabolism and those that are not soluble and stable at gastric $\mathrm{pH}$ are not suitable candidates to be formulated as floating dosage forms (Lauritsen 1990).

Cefuroxime axetil (CA) is abroad spectrum $\beta$ lactamase cephalosporin that has well defined pharmacokinetics after intramuscular and intravenous administration in the form of sodium salt (McEvoy 2003 and Wozniak and Hicks 1991). In human, gastrointestinal absorption of cefuroxime is negligible (Ridgway et al. 1991).Cefuroxim (Cefuroximaxetil) an oral prodrug shows a bioavailability of 30 to $40 \%$ when taken on fasting and 5 to $60 \%$ when taken after food (Sommers, et al. 1984; Finn et al. 1987; McEvoy 1994 and Williams and Harding 1984).

The cefuroximaxetil esterase can hydrolyze cefuroxime axetil to the nonabsorbablecefuroxim in the gut lumen and is therefore, suspected as a possible cause of incomplete bioavailability (Harding 1990) which suggests an absorption mechanism through the mucosa with limited capacity. CA has saturation kinetics that could be overcome by slow release of drug from the formulation, by incorporating the drug in a sustained drug delivery system. Moreover, Cefuroxime axetil has higher absorption in the proximal region of GI tract and poor absorption, as well as antibiotic associated colitis, when a large amount of drug entered the colon. This suggests it as an ideal candidate for a gastroretentive drug delivery system that prolong the gastric residence time of the dosage form, giving controlled drug release in the upper GI tract, where absorption of cefuroxime is well defined.

\section{MATERIALS AND METHODS}

Cefuroxime axetil was a generous gift sample from Alkem laboratories, Mumbai (India). Methocel K15M and E5LV were also gift samples from Mecleods lab, Mumbai. All other chemicals used were of analytical grade.

\section{Preparation of floating tablets}

Cefuroxime axetil loaded floating tablets were prepared by direct compression using Methocel $\mathrm{K} 15 \mathrm{M}$ alone or in combination with E5LV as matrix former and sodium bicarbonate as floating agents. Lactose being water soluble filler was used to maintain the constant tablet weight as well as to counter balance the poor water solubility of drug. Various ingredients (in $\mathrm{mg}$ ) used in different formulations of gastroretentive tablets are presented in the Table 1. Appropriate amounts of the mixture were accurately weighed for the preparation of each tablet.The powder blend was then lubricated with magnesium stearate $(1 \%)$ and compressed by a 10 station rotary tablet punching machine (RimekMinipress I, Ahemadabad, India) using $12 \mathrm{~mm}$ flat face punch. All the prepared formulations were stored in airtight containers at room temperature for further studies.

\section{In vitro buoyancy study}

The in vitro buoyancy was determined by floating lag time, as per the method described by Rosa et al. (1994). The tablets were placed in a $100-\mathrm{mL}$ beaker containing $0.1 \mathrm{~N} \mathrm{HCl}$. The time required for the tablet to rise to the surface and float was determined.

\section{Study of release profile}

The release of CA from the floating tablets was determined by using Dissolution Tester USP XXII. The dissolution test was performed using $900 \mathrm{ml}$ $0.1 \mathrm{~N} \mathrm{HCl}$ solution at $37 \pm 0.5^{\circ} \mathrm{C}$ and the paddles were rotated at $100 \mathrm{rpm}$. At every $1 \mathrm{~h}$ interval, 1.0 $\mathrm{ml}$ of aliquot was withdrawn from the dissolution medium and it was replaced with fresh medium to maintain the volume as constant. The samples were filtered and diluted to suitable concentrations with $0.1 \mathrm{~N} \mathrm{HCl}$ solutions. The absorbance of the solutions was measured at $277.6 \mathrm{~nm}$ for CA with a UV Visible double beam spectrophotometer (Thermo, USA). Cumulative percentage drug release was calculated using an equation obtained from standard curve. The times for 50and $80 \%$ drug release were calculated based on the 
Korsmeyer and Peppas model (Korsmeyer et al. 1983).

\section{Release profile analysis}

For theoretical analysis of dissolution profile of all the batches, the following mathematical model were used: zero order kinetics, first order kinetics (Wagner 1969), Higuchi's square root of time equation (Higuchi 1961), Hixson-Crowell cube root equation (Hixson and Crowell 1931) Korsmeyer and Peppas equations to ascertain the kinetic modeling of drug release.

\section{RESULT AND DISCUSSION}

CA exhibits broad spectrum of activities against gram-positive and Gram-negative microorganism. CA in amorphous form (purities 95\%) has a higher bioavailability than the crystalline form with adequate chemical stability (Somani, et al. 2001). To minimize conversion of amorphous drug into crystalline state, tablets were prepared by direct compression technology (Table 1).

$\underline{\text { Table } 1 \text { - Composition of different formulations }(\mathrm{mg}) \text { of floating tablet. }}$

\begin{tabular}{lccccccccc}
\hline \multirow{2}{*}{ Ingredients } & \multicolumn{10}{c}{ Formulations } & \multicolumn{1}{c}{ AH8 } & AH9 \\
\cline { 2 - 10 } & AH1 & AH2 & AH3 & AH4 & AH5 & AH6 & AH7 & AH8 \\
\hline Cefuroxime axetil & 300 & 300 & 300 & 300 & 300 & 300 & 300 & 300 & 300 \\
HPMC K15M & 60 & 60 & 60 & 54 & 54 & 54 & 48 & 48 & 48 \\
HPMC E5LV & - & - & - & 6 & 6 & 6 & 12 & 12 & 12 \\
Sodium Bicarbonate & 75 & 75 & 75 & 75 & 75 & 75 & 75 & 75 & 75 \\
Magnesium Stearate & 6 & 6 & 6 & 6 & 6 & 6 & 6 & 6 & 6 \\
D.C.Lactose & 159 & 156 & 153 & 159 & 156 & 153 & 159 & 156 & 153 \\
Sodium Laurayl Sulfate & - & 3 & 6 & - & 3 & 6 & - & 3 & 6 \\
\hline
\end{tabular}

\section{Release Rate Analysis}

After pre-formulary of dissolution study, the drug release rate was analyzed by various mathematical models.

The drug release rate from one component polymeric system (AH1) was slow and, only $64.45 \pm 1.78$ drug was released in $12 \mathrm{~h}$ (Fig. 4). Hence, different ratio of HPMC K15M and HPMC E5LV were fabricated in order to optimize the release pattern. In two compartment polymeric system $\mathrm{AH} 4$ and $\mathrm{AH} 7$, drug release rate increased with increasing E5LV concentration. The lower release rate in one compartment system, (AH1) could be explained by thes welling of polymer after it absorbed water. Higher amount of HPMC $\mathrm{K} 15 \mathrm{M}$ imbibed with water caused greater degree of swelling (thicker gel layer formulation). Thisin turn, increased the tortuosity and length of drug diffusion path, thus decreasing the amount of drug release (Fig. 1). The polymeric system with higher concentration of HPMC E5LV yielded faster drug release rate due to gradual decrease in viscosity, caused erosion rather than swellingand thus, decreasing the diffusion pathlength (Dortunc and Gunal 1997).

To counter balance the poor solubilization of CA, 0.5 and $1 \%$ concentration of anionic surfactant, sodium lauryl sulfate was used in different polymeric blend. Significant difference $(p<0.5)$ in drug release rate was found in different concentration of SLS of same polymeric concentration (Fig. 2 and 3).

Drug dissolution was increased with the increased concentration of SLS up to $0.5 \%$; further increasing the SLS concentration lowered the dissolution at $1 \%$ level (Fig. 4). This might be due to the solubilization effect of SLS at $0.5 \%$ level, which was not observed at $1 \%$ level, because of the formation of micelle. 


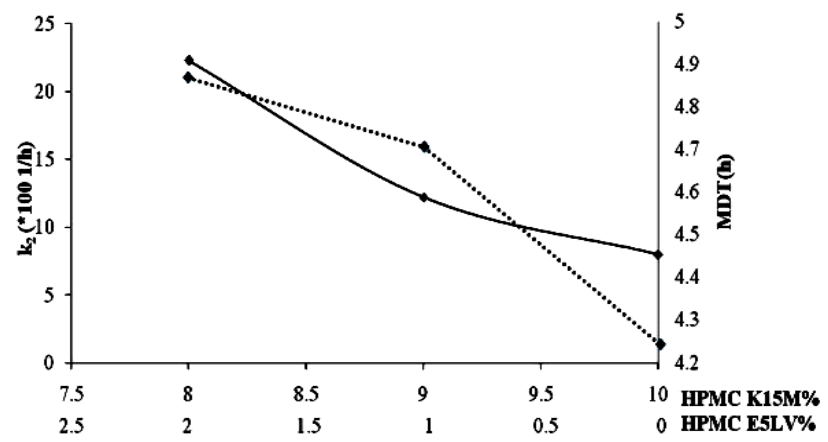

Figure 1- Relationship between release rate of CA and content of HPMC (solid line release rate $\mathrm{k}_{2}$, dashed line MDT).

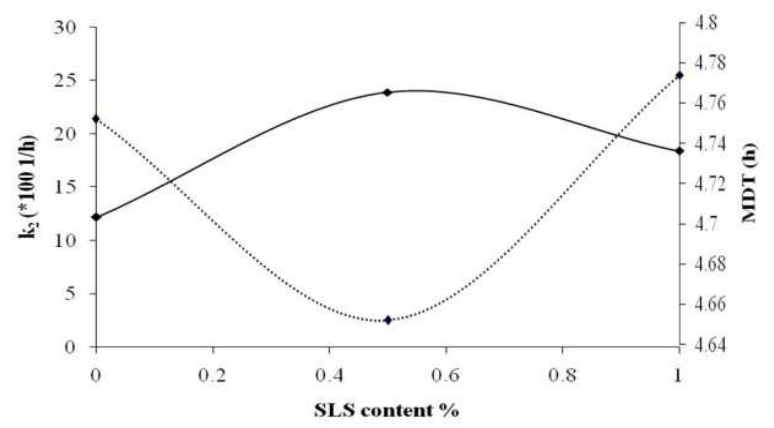

Figure 2- Relation between release rate and blend of SLS at 9\% HPMC K15MHPMC (solid line release rate $\mathrm{k}_{2}$, dashed line MDT).

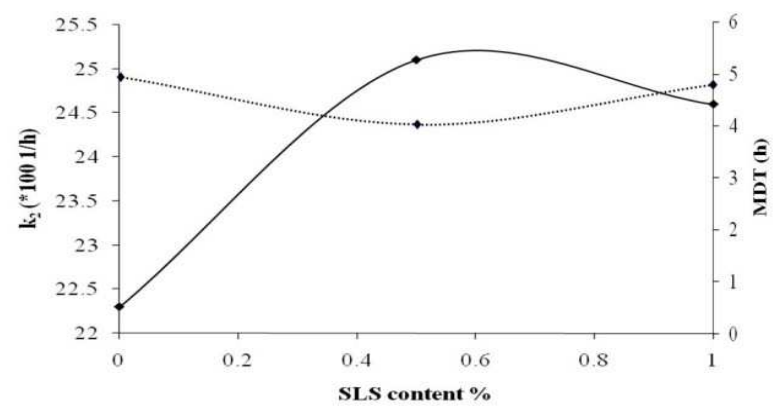

Figure 3 - Relation between release rate and blend of SLS content at 8\% HPMC K15M.

$\boldsymbol{A}$

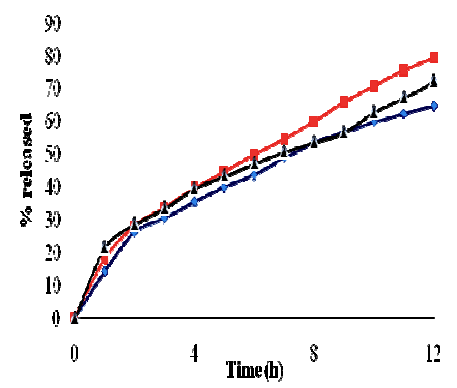

$\boldsymbol{B}$

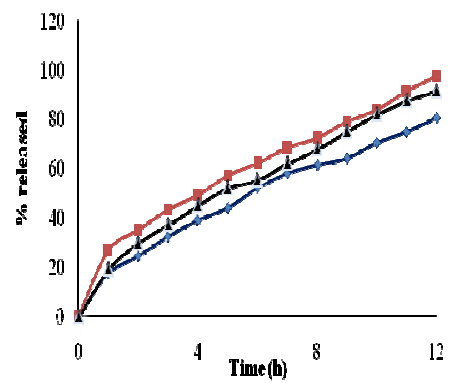

$C$

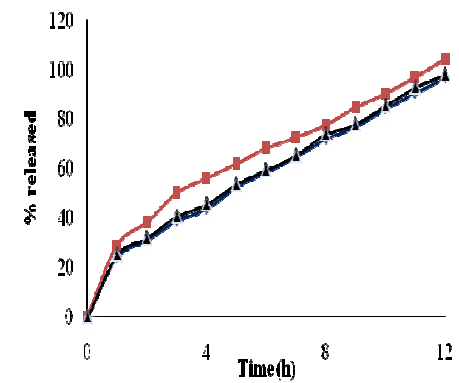

Figure 4 - Release of CA from various systems.

A. Release of CA from various formulations containing $10 \%$ Methocel $\mathrm{K} 15 \mathrm{M}$ with $\neg-0 \%,-0.5 \%,-\mathbf{A}-1 \%$ SLS.

B. Release of CA from various formulations containing 9\% Methocel K15M with $-0 \%,-0.5 \%$. $-1 \%$ SLS.

C. Release of CA from various formulations containing $8 \%$ Methocel K15M with $\longrightarrow 0 \%,-0.5 \%,-\mathbf{A}-1 \%$ SLS. 


\section{Release mechanism analysis}

Based on the kinetics model, discussed previously, the best linear relation was shown to be Higuchi's square root of time equation. The value of release exponent (n) were calculated from Korsemeyer and Peppas equation .The " $n$ " value all formulation within 0.49 to 0.59 (Table 2), indicated anomalous transport, which was co dominated by both diffusion and polymer erosion in the release mechanism.

Table2 - Floating lag time and various release parameters for floating table.

\begin{tabular}{cccccccccc}
\hline \multirow{2}{*}{$\begin{array}{c}\text { Batches } \\
\text { Code }\end{array}$} & $\begin{array}{c}\text { Floating Lag } \\
\text { time (Minute) }\end{array}$ & \multirow{2}{*}{$\begin{array}{c}\mathbf{t}_{\mathbf{5 0} \%} \\
\mathbf{h r r s})\end{array}$} & \multicolumn{2}{c}{ Zero order } & \multicolumn{2}{c}{ Higuchi } & \multicolumn{2}{c}{ Korsmeyer } & \multirow{2}{*}{ MDT } \\
\cline { 5 - 9 } & & $\mathbf{K}_{\mathbf{0}}$ & $\mathbf{R}^{\mathbf{2}}$ & $\mathbf{\mathbf { K } _ { \mathbf { h } }}$ & $\mathbf{R}^{\mathbf{2}}$ & $\mathbf{n}$ & $\mathbf{R}^{\mathbf{2}}$ & \\
\hline AH1 & 15 & 7.3 & 4.879 & 0.94 & 19.39 & 0.995 & 0.585 & 0.989 & 4.239 \\
AH2 & 15 & 5.6 & 5.921 & 0.957 & 23.28 & 0.992 & 0.589 & 0.997 & 4.596 \\
AH3 & 25 & 6.5 & 4.971 & 0.934 & 19.79 & 0.992 & 0.48 & 0.99 & 4.522 \\
AH4 & 14 & 5.7 & 5.991 & 0.961 & 23.48 & 0.99 & 0.604 & 0.997 & 4.752 \\
AH5 & 2 & 3.7 & 6.982 & 0.953 & 27.5 & 0.99 & 0.525 & 0.991 & 4.652 \\
AH6 & 28 & 4.7 & 6.944 & 0.969 & 27.03 & 0.984 & 0.62 & 0.996 & 4.931 \\
AH7 & 20 & 4.3 & 7.12 & 0.974 & 27.79 & 0.98 & 0.581 & 0.983 & 4.774 \\
AH8 & 21 & 3.2 & 7.48 & 0.931 & 28.43 & 0.996 & 0.501 & 0.995 & 4.025 \\
AH9 & 24 & 4.05 & 7.208 & 0.967 & 28.09 & 0.984 & 0.564 & 0.986 & 4.79 \\
\hline
\end{tabular}

\section{Selection of optimized batch}

The comparative dissolution results of the different batches were analyzed so as to get the optimized formulation. Dissolution profile of formulations contained only HPMC K15M, such as, $\mathrm{AH} 1, \mathrm{AH} 2, \mathrm{AH} 3$, were less in desired time periods. Dissolution could be enhanced by incorporation of low viscosity polymer HPMCE5LValong with K15M from AH4 to AH9. Release profile of AH4 was slower (less than $80 \%$ ) even if $1 \%$ HPMC was replaced with E5LV. Solubility increased with increased SLS up to $5 \%$ then decreased. Thus, the drug release rate was increased in AH5 up to 15 to $20 \%$, but decreased the release rate in case of $1 \%$ SLS level of AH6.

Similar type of release pattern was observed in case of formulation containing 8\% HPMC E5LV; but at 5\%level of SLS, 97.54 \pm 0.68 drug was released in $11 \mathrm{~h}$ (Fig. 4). The time required for $50 \%$ drug release $\left(\mathrm{t}_{50 \%}\right)$ and mean dissolution time
(MDT) (Mockel, et al. 1993) of formulation AH4 to AH9 was determined and compared to get the optimized formulation (Table 2). Batch AH6 had highest $\mathrm{t}_{50 \%}$ and MDT value.

When dissolution data of AH6 was plotted in terms of the Hixson-Crowell cube root law, the compliance of this formulation to the equation indicated a change in surface area and diameter of tablets, due to the progressive dissolution of the matrix as a function of time (Fig. 5).

\section{Drug excipient interaction study}

Drug excipient interaction plays a vital role in the release of drug from formulation. FTIR techniques have been used study the physical and chemical interactions between the drug and excipient used. In the present study, no chemical interaction between $\mathrm{CA}$ and excipients used was found (Fig. $6)$.

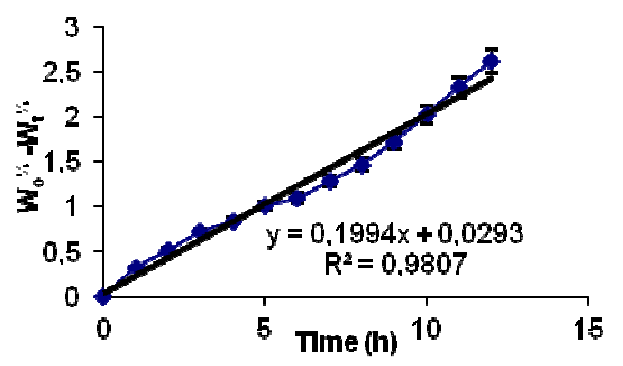

Figure 5 - Hixon Crowell release profiles of formulation AH6 $(n=6$, mean \pm SD). 


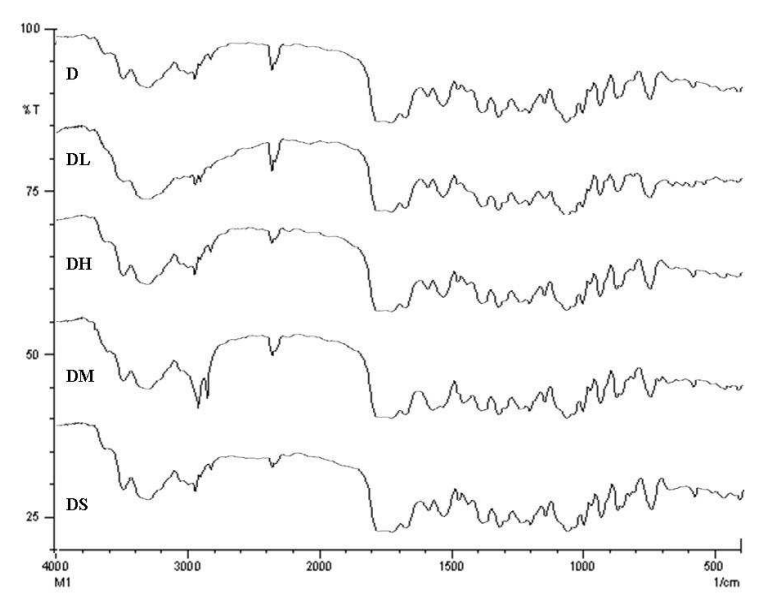

Figure 6 - FT-IR Spectra of Pure Cefurixime axetil (D), Solid Admixture of CA with lactose (DL), CA with HPMC (DH), CA with Magnesium Stearate (DM) and CA with SLS (DS).

\section{CONCLUSION}

From the results, it could be concluded that for the development of controlled release dosage from poorly water soluble drug, polymer blends of different viscosity grade of HPMC and presence of surfactant were useful which imparted hydrophilic environment and wettability to molecules of drug which led more uniform drug release.

\section{ACKNOWLEDGEMENT}

Authors are thankful to Alkem Lab. (Mumbai, India) for providing gift sample of cefuroxime axetil.

\section{REFERENCES}

Deshpande AA, Rhodes CT, Shah NH and Malick AW. Controlled-release drug delivery systems for prolonged gastric residence: an overview. Drug Dev. Ind.Pharm. 1996; 22:531-539.

DortuncB, and Gunal N. Release Characteristics from swallable HPMC matrix tablets. Drug Dev Ind. Pharm. 1997; 23:1245-1249.

Finn A, StraugunA, Meyer M, and Chubb J. Effect of dose and food on the bioavailability of cefuroxime axetil. Biopharm Drug Dispos. 1987; 8: 519-526.

Harding SM. Comparativepharmacokinetics of tablets and suspension. Res ClinForums. 1990: 12: 23-29.
Higuchi T. Rate of release of medicaments from ointment bases containing drugs in suspension. $J$ Pharm Sci.1961; 50:874-875.

Hixson AW and Crowell JH.Dependence of reaction velocity upon surface and agitation.Ind. Eng. Chem.1931; 23:923-931.

Iannuccelli V,Coppi G,Bernabei MT, and Cameroni R. Air compartments multiple unit system for prolonged gastric residence. Part I. Formulation study.Intl. J. Pharm. 1998; 174:47-54.

Korsmeyer R,Gurny R, and Peppas N. Mechanisms of solute release from porous hydrophilic polymers. Intl. J. Pharm. 1983; 15: 25-35.

Lauritsen K. Clinical pharmacokinetics of drugs used in the treatment of gastrointestinal diseases.ClinPharmacokinet. 1990; 19: 94-125.

McEvoy GK, ed. Cephalosporins, cefuroxime sodium and cefuroxime axetil. In: Bethesda, MD,AHFS Drug Information. American Society of Hospital Pharmacists Inc; Wisconsin Avenue, Bethesda: 1994, p.152-159.

McEvoy GK. ed. Cephalosporins: cefuroxime sodium and cefuroxime axetil. In: Bethesda, MD:AHFS Drug Information. American Society of Hospital Pharmacists Inc;Wisconsin Avenue, Bethesda: 2003, p. 223-231.

Menon A, Ritschel WA and Sakr A. Development and evaluation of a monolithic floating dosage form for furosemide. J. Pharm. Sci.1994; 83: 239-245.

MockelJE and Lippold BC.Zero order release from hydrocolloid matrices. Pharm Res.1993; 10:1066Y1070. 
MoesAJ. Gastro retentive dosage forms.Crit. Rev. Ther. Drug Carrier.Syst. 1993; 10: 143-159.

Ozdemir N, Ordu S, and Ozkan Y. Studies of floating dosage forms of furosemide: in vitro and in vivo evaluation of bilayer tablet formulations. Drug Dev. Ind. Pharm. 2000; 26: 857-866.

PonchelG, and Irache JM.Specific and non-specific bioadhesive particulate system for oral delivery to the gastrointestinal tract.Adv. Drug Del. Rev. 1998; 34: 191-219.

Ridgway E, Stewart K, Rai G, Kelsey MC and Bielawska C. The pharmacokinetics of cefuroxime axetil in the sick elderly patient. $J$ Antimicrob Chemother. 1991; 27: 663-668.

Rosa M, Zia H, and Rhodes T. Dosing and testing invitro of a bioadhesive and floating drug delivery system for oral application. Intl. J. Pharm. 1994; 105: 65-70.

Santus G, Lazzasrini $\mathrm{G}$ and Bottoni G. An in vitroinvivo investigation of oral bioadhesive controlled release furosemide formulations. EurJ.Pharm. Biopharm. 1997; 44: 39-52.
Somani JK, Bhusan I, and Sen H. Bioavailable dosage form of cefuroxime axetil, US Pat. 2001; 323(6): 193.

Sommers D, VanWyk K, Schoeman H and Moncrieff J. Influence of food and reduced gastric acidity on the bioavailability of bacampicillin and cefuroxime axetil. Br J Clin Pharmacol. 1984; 18: 535-539.

Wagner JG. Interpretation of percent dissolved-time plots derived from in vitro testing of conventional tablets and capsules. J. Pharm. Sci. 1969; 58: 1253 1257.

Williams $\mathrm{P}$ and Harding SM, The absolute bioavailability of oral cefuroxime axetil in male and female volunteers after fasting and after food. $J$ Antimicrob Chemother. 1984; 13: 191-196.

Wozniak T and Hicks J. Cefuroxime sodium. In: Florey K, ed. Analytical Profiles of Drug Substances. vol.20. San Diego, CA: Academic Press; 1991.p. 209-237.

Received: August 23, 2010; Revised: September 09, 2011; Accepted: December 28, 2011 
PÁGINA

EM

BRANCO 